\title{
ASTEROSEISMOLOGY OF HYBRID PULSATORS MADE POSSIBLE: SIMULTANEOUS MOST SPACE PHOTOMETRY AND GROUND-BASED SPECTROSCOPY OF $\gamma$ PEG ${ }^{*}$
}

\author{
G. Handler ${ }^{1}$, J. M. Matthews ${ }^{2}$, J. A. Eaton ${ }^{3}$, J. Daszyńska-Daszkiewicz ${ }^{4}$, R. Kuschnig ${ }^{1}$, H. Lehmann ${ }^{5}$, E. Rodríguez ${ }^{6}$, \\ A. A. Pamyatnykh ${ }^{1,7,8}$, T. Zdravkov ${ }^{7}$, P. Lenz $^{1}$, V. Costa ${ }^{6}$, D. Díaz-Fraile ${ }^{6}$, A. Sota ${ }^{6}$, T. Kwiatkowski ${ }^{9}$, \\ A. Schwarzenberg-CZerny ${ }^{7}$, W. BorczyK ${ }^{9}$, W. Dimitrov ${ }^{9}$, M. Fagas ${ }^{9}$, K. Kamiński $^{9}$, A. RożeK ${ }^{9}$, F. van WyK ${ }^{10}$, \\ K. R. Pollard ${ }^{11}$, P. M. Kilmartin ${ }^{11}$, W. W. Weiss ${ }^{1}$, D. B. Guenther ${ }^{12}$, A. F. J. Moffat ${ }^{13}$, S. M. Rucinski ${ }^{14}$, D. D. Sasselov ${ }^{15}$, \\ AND G. A. H. WALKER ${ }^{16}$ \\ ${ }^{1}$ Institut für Astronomie, Universität Wien, Türkenschanzstrasse 17, A-1180 Wien, Austria \\ 2 Department of Physics and Astronomy, University of British Columbia, 6224 Agricultural Road, Vancouver, BC V6T 1Z1, Canada \\ ${ }^{3}$ Center of Excellence in Information Systems, Tennessee State University, Nashville, TN 37209, USA \\ ${ }^{4}$ Instytut Astronomiczny, Uniwersytet Wrocławski, ul. Kopernika 11, 51-622 Wrocław, Poland \\ ${ }^{5}$ Thüringer Landessternwarte Tautenburg, 07778 Tautenburg, Germany \\ ${ }^{6}$ Instituto de Astrofísica de Andalucia, CSIC, P.O. Box 3004, 18080 Granada, Spain \\ ${ }^{7}$ Copernicus Astronomical Center, Bartycka 18, 00-716 Warsaw, Poland \\ ${ }^{8}$ Institute of Astronomy, Russian Academy of Sciences, Pyatnitskaya Str. 48, 109017 Moscow, Russia \\ ${ }^{9}$ Astronomical Observatory, Adam Mickiewicz University, Słoneczna 36, 60-286 Poznan, Poland \\ ${ }^{10}$ South African Astronomical Observatory, P.O. Box 9, Observatory 7935, South Africa \\ ${ }^{11}$ Department of Physics and Astronomy, University of Canterbury, Private Bag 4800, Christchurch, New Zealand \\ ${ }^{12}$ Department of Astronomy and Physics, St. Mary's University, Halifax, NS B3H 3C3, Canada \\ ${ }^{13}$ Départment de physique, Université de Montréal, C. P. 6128, Succ. Centre-Ville, Montréal, QC H3C 3J7, Canada \\ ${ }^{14}$ Department of Astronomy and Astrophysics, University of Toronto, 50 St. George Street, Toronto, ON M5S 3H4, Canada \\ ${ }^{15}$ Astronomy Department, Harvard University, 60 Garden Street, Cambridge, MA 02138, USA \\ 161234 Hewlett Place, Victoria, BC V8S 4P7, Canada \\ Received 2009 April 21; accepted 2009 May 7; published 2009 May 21
}

\begin{abstract}
We have acquired simultaneous high-precision space photometry and radial velocities of the bright hybrid $\beta$ Cep/Slowly Pulsating B pulsator $\gamma$ Peg. Frequency analyses reveal the presence of six gravity (g) modes of high radial order together with eight low-order $\beta$ Cep oscillations in both data sets. Mode identification shows that all pulsations have spherical degrees $\ell=0-2$. An $8.5 M_{\odot}$ model reproduces the observed pulsation frequencies; all theoretically predicted modes in the $\beta$ Cep domain are detected. We suggest, contrary to previous authors, that $\gamma$ Peg is a single star; the claimed orbital variations are due to g-mode pulsation. $\gamma$ Peg is the first hybrid pulsator for which a sufficiently large number of high-order g modes and low-order pressure (p) and mixed modes have been detected and identified to be usable for in-depth seismic modeling.
\end{abstract}

Key words: binaries: spectroscopic - stars: early-type - stars: individual ( $\gamma$ Peg) - stars: oscillations - stars: variables: other

\section{INTRODUCTION}

The bright $(V=2.8)$ B2 IV star $\gamma$ Peg was recognized as a pulsating variable of the $\beta$ Cep class more than 50 years ago (McNamara 1953), and it was believed to be singly periodic until recently. Chapellier et al. (2006) studied the star spectroscopically and demonstrated its multiperiodicity. These authors also examined the claim that $\gamma$ Peg is a spectroscopic binary and deduced an eccentric $(e=0.62) 370.5$ day orbit. This orbital solution was disputed by Butkovskaya \& Plachinda (2007) who suggested that the orbital eccentricity was spurious and caused by outbursts similar to those of Be stars, and favored an orbital period near $6.8 \mathrm{~d}$, in accordance with the original suggestion by Harmanec et al. (1979).

The multiperiodic oscillations of $\gamma$ Peg are highly interesting because they are caused by two different sets of pulsation modes: two frequencies detected by Chapellier et al. (2006) correspond to low-order pressure (p) and gravity (g) modes typical for $\beta$ Cep stars, but the other two are high-order g modes as excited in

\footnotetext{
* Based on data from the MOST satellite, a Canadian Space Agency mission operated by Dynacon, Inc., the University of Toronto Institute of Aerospace Studies, and the University of British Columbia, with assistance from the University of Vienna, Austria.
}

the Slowly Pulsating B (SPB) stars. Indeed, $\gamma$ Peg is located in the overlap region of both types of variables in the HR diagram (see Handler 2009). The frequencies of the two sets of modes are sensitive to the physical conditions in different parts of the stellar interior. Hybrid oscillators therefore offer the possibility to obtain a more complete picture of the physics inside a star using asteroseismology where pulsations act as seismic waves (see, e.g., Dziembowski \& Pamyatnykh 2008 for case studies).

However, the possible binarity of $\gamma$ Peg imposes difficulties. The detection of low-frequency oscillations can be compromised by an inaccurate orbital solution. Photometry does not suffer from this problem (provided the light-time effect is negligible). Consequently, Handler (2009) carried out a multicolor time-series photometric study of $\gamma$ Peg, detected four SPB-type pulsation modes, and confirmed the two modes of $\beta$ Cep type. One of the latter was identified as radial; it would be either the fundamental mode or the first overtone, immediately constraining the mean stellar density. The enormous asteroseismic promise of $\gamma$ Peg motivated us to perform a high-precision photometric and radial velocity study of its pulsations, in the hope to detect a sufficient number of $\mathrm{p}$ and $\mathrm{g}$ modes to sound its interior structure. 


\section{OBSERVATIONS}

$\gamma$ Peg was observed with the MOST satellite (Walker et al. 2003) from 2008 September 16 to October 16. Due to the brightness of the target, the Fabry imaging mode was used, and the data reduction method developed by Reegen et al. (2006) to minimize the effects of stray light was employed. There were over 55,000 data points at a cadence of $30 \mathrm{~s}$ in the original data set. These were summed into $40180.005 \mathrm{~d}$ bins having an rms scatter of 1 mmag per point and an effective Nyquist frequency of $\sim 92 \mathrm{~cd}^{-1}$.

A simultaneous ground-based spectroscopic multisite campaign was organized for pulsational mode identification. The majority of the data originated from the Automatic Spectroscopic Telescope of Tennessee State University (TSU-AST; Eaton \& Williamson 2007), and consisted of 1660 spectra (4900-7100 A) taken over a span of 10 weeks (2008 September 3-November 15) with an effective Nyquist frequency of $\sim 68 \mathrm{~cd}^{-1}$. We have reduced these spectra and extracted radial velocities from 34 lines with the techniques described by Eaton $\&$ Williamson (2007). The external error of these radial velocities is about $0.2 \mathrm{~km} \mathrm{~s}^{-1}$. This high precision results from $\gamma$ Peg having a very sharp lined spectrum for a hot star; Telting et al. (2006) listed $v \sin i=0 \mathrm{~km} \mathrm{~s}^{-1}$ ! Concerning the star's metallicity, Morel et al. (2006) derived $Z=0.0091 \pm 0.0021$ from optical and Daszyńska-Daszkiewicz \& Niemczura (2005) determined $[m / H]=-0.04 \pm 0.08$ from ultraviolet spectra.

Additional spectroscopy was carried out at three observatories, and ground-based time-resolved multicolor photometry was acquired at three more sites. However, this Letter only reports the initial results from the MOST space photometry and TSU-AST radial velocities.

\section{FREQUENCY ANALYSIS}

The heliocentrically corrected data were searched for periodicities using the program Period04 (Lenz \& Breger 2005). Amplitude spectra were computed, compared with the spectral window functions, and the frequencies of the intrinsic and statistically significant peaks in the Fourier spectra were determined. Multifrequency fits with all detected signals were calculated step by step, the corresponding frequencies, amplitudes, and phases were optimized and subtracted from the data before computing residual amplitude spectra, which were then examined in the same way.

This analysis was performed for the MOST and radial velocity data independently. We conservatively only accepted signals that exceeded an amplitude signal-to-noise ratio $(\mathrm{S} / \mathrm{N})$ of 5 in at least one of the data sets and that were prominent in the other. Some steps of this procedure are shown in Figure 1. Fourteen independent signals were detected; the agreement between the photometric and radial velocity measurements is remarkable. The residual amplitude spectrum after this solution was featureless for the radial velocities. Some peaks in the residual MOST data remained, but to err on the side of caution we did not consider them to be intrinsic to $\gamma$ Peg.

The frequencies of all these signals are consistent within the errors between the two data sets. We have determined weighted mean values of those frequencies by computing the formal uncertainties (Montgomery \& O'Donoghue 1999) in the individual data sets and then applying their inverse squared as the weight. With the resulting frequencies fixed, we have recalculated the amplitudes, phases, and amplitude S/Ns and list the results in Table 1.

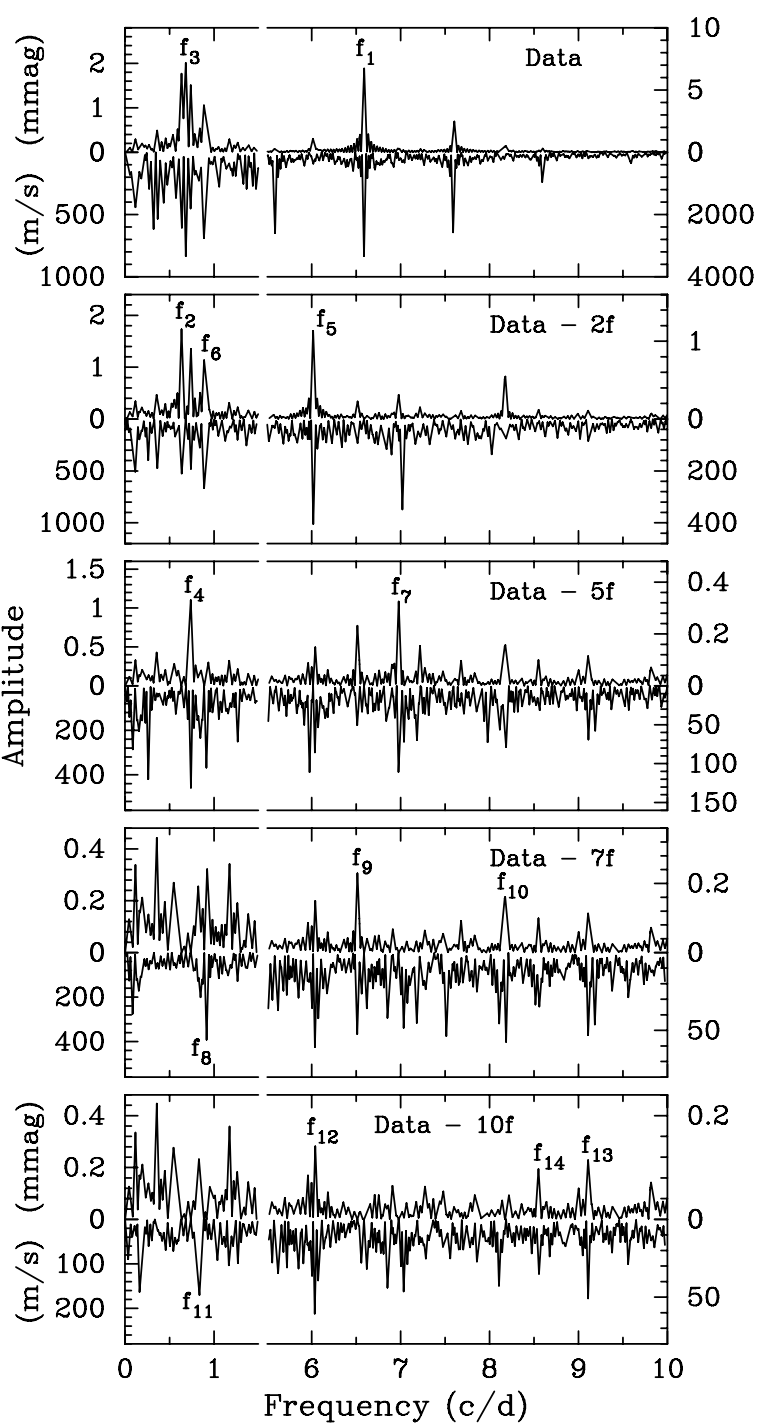

Figure 1. Amplitude spectra of our $\gamma$ Peg data with consecutive prewhitening. The photometric data are compared with the radial velocity measurements (reverted graphs). The frequency regions $1.5-5.5$ and $>10 \mathrm{~cd}^{-1}$ are not shown as they contain no intrinsic signals. The frequencies $f_{1}-f_{6}$ have been assigned for consistency with Handler (2009). The apparent $1 \mathrm{~cd}^{-1}$ sidelobe of $f_{1}$ in the $M O S T$ data is an alias peak originating from the orbital frequency.

All but the two closest frequencies are resolved within our data set. Their difference $f_{12}-f_{5}=0.0112 \mathrm{~cd}^{-1}$ is $82 \%$ of the time resolution of the radial velocity data. Since a significant peak remains after prewhitening $f_{5}$ we accept $f_{12}$, but caution that the parameters of these two variations in Table 1 may have systematic errors.

\section{DISCUSSION}

We start by examining previous claims that $\gamma$ Peg is a spectroscopic binary. Our radial velocities were acquired in a period of time where the orbital solution by Chapellier et al. (2006) predicts a change in radial velocity of about $20 \mathrm{~km} \mathrm{~s}^{-1}$. Our nightly mean radial velocities are constant within $\pm 0.3 \mathrm{~km} \mathrm{~s}^{-1}$. Butkovskaya \& Plachinda (2007) claimed an orbit with a $6.816 \mathrm{~d}$ period and about $0.8 \mathrm{~km} \mathrm{~s}^{-1}$ radial velocity amplitude. Again, this is inconsistent with our data. Instead, the $1 \mathrm{~cd}^{-1}$ alias of this "orbital" period lies well within the domain of the g-mode frequencies $\left(1-1 / 6.816 \mathrm{~d}=0.8533 \mathrm{~cd}^{-1}\right)$; the strongest $\mathrm{g}$-mode pulsations have radial velocity amplitudes 
Table 1

Multifrequency Solution for our Photometric and Radial Velocity Data

\begin{tabular}{lcccrrr}
\hline \hline ID & $\begin{array}{c}\text { Frequency } \\
\left(\mathrm{cd}^{-1}\right)\end{array}$ & $\begin{array}{c}\text { Amplitude } \\
(\mathrm{mmag})\end{array}$ & $\begin{array}{c}\text { Amplitude } \\
\left(\mathrm{km} \mathrm{s}^{-1}\right)\end{array}$ & $\begin{array}{c}(\mathrm{S} / \mathrm{N})_{\text {phot }} \\
(\mathrm{S} / \mathrm{N})_{\mathrm{RV}}\end{array}$ & $\begin{array}{c}\phi_{\text {Vrad }}-\phi_{\text {MOST }} \\
(\mathrm{rad})\end{array}$ \\
\hline$f_{1}$ & $6.58974 \pm 0.00002$ & 6.59 & 3.359 & 300.9 & 327.9 & $4.318 \pm 0.004$ \\
$f_{2}$ & $0.63551 \pm 0.00010$ & 1.70 & 0.500 & 21.3 & 17.5 & $2.189 \pm 0.019$ \\
$f_{3}$ & $0.68241 \pm 0.00007$ & 1.99 & 0.736 & 24.8 & 26.0 & $2.280 \pm 0.015$ \\
$f_{4}$ & $0.73940 \pm 0.00010$ & 1.23 & 0.522 & 15.4 & 18.6 & $2.481 \pm 0.023$ \\
$f_{5}$ & $6.01616 \pm 0.00014$ & 1.14 & 0.358 & 45.0 & 34.4 & $4.55 \pm 0.03$ \\
$f_{6}$ & $0.88550 \pm 0.00007$ & 0.90 & 0.723 & 11.3 & 25.9 & $3.18 \pm 0.03$ \\
$f_{7}$ & $6.9776 \pm 0.0005$ & 0.33 & 0.095 & 16.3 & 9.5 & $4.40 \pm 0.10$ \\
$f_{8}$ & $0.91442 \pm 0.00011$ & 0.51 & 0.464 & 6.4 & 16.6 & $3.79 \pm 0.05$ \\
$f_{9}$ & $6.5150 \pm 0.0008$ & 0.21 & 0.063 & 9.4 & 6.3 & $4.78 \pm 0.15$ \\
$f_{10}$ & $8.1861 \pm 0.0008$ & 0.18 & 0.064 & 9.2 & 6.7 & $4.16 \pm 0.17$ \\
$f_{11}$ & $0.8352 \pm 0.0003$ & 0.27 & 0.180 & 3.4 & 6.3 & $2.61 \pm 0.09$ \\
$f_{12}$ & $6.0273 \pm 0.0005:$ & 0.33 & 0.112 & 12.4 & 10.3 & $4.81 \pm 0.10$ \\
$f_{13}$ & $9.1092 \pm 0.0012$ & 0.12 & 0.041 & 5.9 & 4.5 & $3.97 \pm 0.26$ \\
$f_{14}$ & $8.552 \pm 0.002$ & 0.10 & 0.027 & 5.0 & 3.2 & $4.06 \pm 0.34$ \\
\hline
\end{tabular}

Notes. The formal errors on the photometric amplitudes are $\pm 0.02 \mathrm{mmag}$; the $1 \sigma$ errors on the radial velocity amplitudes are $\pm 0.007 \mathrm{~km} \mathrm{~s}^{-1} \cdot f_{12}$ has been marked with a colon because it is not fully resolved from $f_{5}$.

of $0.7 \mathrm{~km} \mathrm{~s}^{-1}$. We conclude that $\gamma$ Peg is not a $6.8 \mathrm{~d}$ spectroscopic binary. The cause of the sporadic radial velocity changes remains to be understood. The possibility of Be-star outbursts (Butkovskaya \& Plachinda 2007) seems remote given that $\gamma$ Peg likely rotates intrinsically slowly, unlike the Be stars (e.g., Porter 1996).

According to the position of $\gamma$ Peg in the HR diagram determined by Handler (2009), all signals with frequencies below $1 \mathrm{~cd}^{-1}$ are due to high-order $\mathrm{g}$ modes; the remaining variations are caused by low-order $\mathrm{p}$ and mixed modes. Handler (2009) identified $f_{1}$ as a radial mode and argued that $f_{5}$ is a dipole mode; these are the only two modes with reasonably secure identifications. From comparison with pulsation models, this author concluded that models in just two small domains of parameter space in the HR diagram are consistent with these mode identifications and with the frequency values. In case $f_{1}$ is the radial fundamental mode, models around $8.5 M_{\odot}$ match the observations. If $f_{1}$ corresponded to the first radial overtone, models with masses around $9.6 M_{\odot}$ reproduce $f_{1}$ and $f_{5}$. All models in this Letter and by Handler (2009) were computed with the Warsaw-New Jersey stellar evolution and pulsation code (e.g., see Pamyatnykh et al. 1998 for a description), using OP opacities, the Asplund et al. (2004) element mixture, and providing linear nonadiabatic model frequencies. No rotation or convective core overshooting was included in the models for simplicity of this preliminary model fitting. Figure $2 \mathrm{com}-$ pares the additional $\ell=0-2 \beta$ Cep-type pulsation frequencies predicted by the two models with our new observations.

All newly detected pulsation frequencies are explained by the $8.5 M_{\odot}$ model (upper panel of Figure 2). At first sight, there are two small inconsistencies: the close doublet $f_{5} / f_{12}$ only has one theoretical counterpart, and $f_{13}$ is not well matched by the theoretical value. The doublet can be explained by possible rotational splitting at $v_{\text {rot }} \approx 3 \mathrm{~km} \mathrm{~s}^{-1}$, consistent with the very low $v \sin i$ of the star. The mismatch for $f_{13}$ may be more of a problem, but it would not be new: in their analysis of the pulsation spectrum of $v$ Eri, Pamyatnykh et al. (2004) noted the same problem for the highest-frequency $\mathrm{p}$ mode $(\ell=1$, $\mathrm{p}_{2}$ ) observed. Interestingly, in the present model, $f_{13}$ would also correspond to the $\ell=1, \mathrm{p}_{2}$ mode and even the size of the frequency mismatch is similar. It can be suspected that this

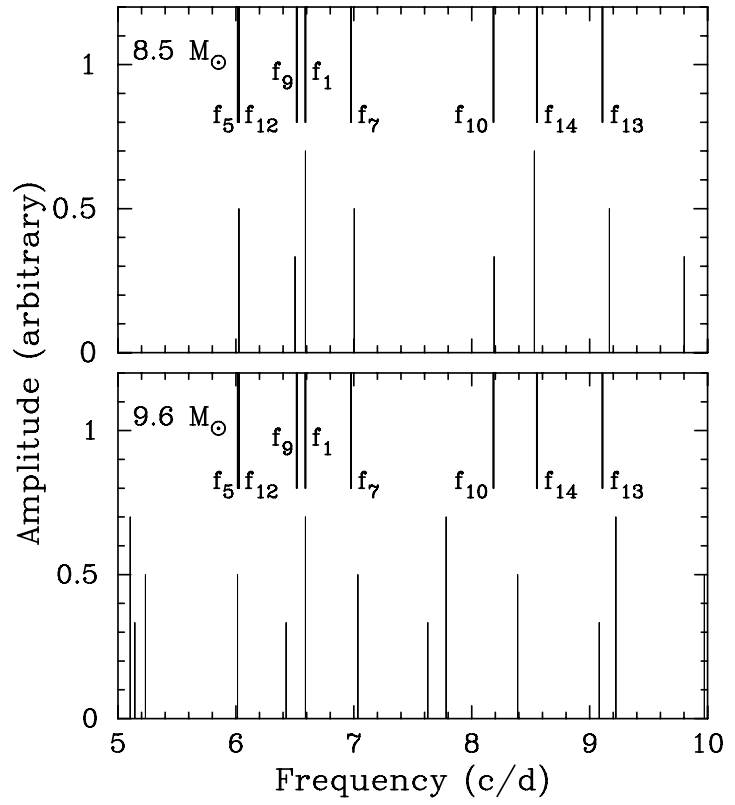

Figure 2. Comparison of theoretically predicted pulsation frequencies of two models fitting $f_{1}$ and $f_{5}$. The model frequencies are plotted at the bottom of the panels, with amplitudes of 0.7 (radial modes), 0.5 (dipole modes), and 0.33 (quadrupole modes). The observed frequencies protrude from the top of the panels.

mismatch originates from inadequate physics in the models that can be improved through asteroseismology.

The 9.6 $M_{\odot}$ model (lower panel of Figure 2) results in poorer agreement between the observed and theoretical frequencies, although the number of theoretically predicted modes in the domain of excited frequencies is larger. In particular, only one theoretical $\ell=1$ mode is available to match $f_{10}$ and $f_{14}$. One would need to invoke rather fast rotation to explain both modes, but in this case $\gamma$ Peg would be viewed close to poleon, which would cause heavy geometrical cancellation of just these pulsations. We conclude that, in all likelihood, $\gamma$ Peg is a $\sim 8.5 M_{\odot}$ star oscillating with a dominant radial fundamental mode. In this case, all the theoretically predicted $\ell=0-2 \beta$ Cep-type pulsation modes are observed. 

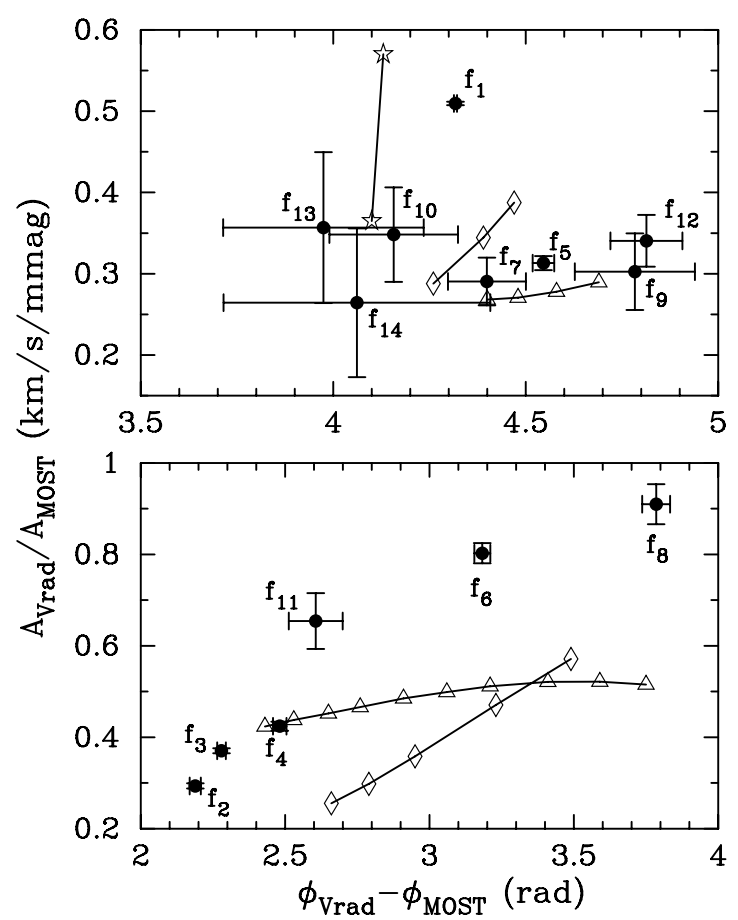

Figure 3. Observed (full circles with error bars) and theoretically predicted radial-velocity to photometric amplitude ratios and phase shifts for the 14 signals detected. The upper panel shows the $\beta$ Cep pulsations and the lower panel the SPB oscillation modes. The star symbols show the theoretical locations of radial modes in this diagram, the diamonds represent dipole modes and the triangles stand for quadrupole modes. Only model modes with frequencies in the observed domains are plotted.

The amplitudes and phases of the oscillations can be used to type the pulsation modes. We have computed theoretical radial velocity to light amplitude ratios and phase differences with for the MOST bandpass. The $8.5 M_{\odot}$ model was used together with static atmospheres (Kurucz 2004) with a metallicity parameter $[\mathrm{m} / \mathrm{H}]=0.0$ and a microturbulence velocity $\xi_{t}=2 \mathrm{~km} \mathrm{~s}^{-1}$. The comparison of the theoretical and observed amplitude ratios and phase shifts is shown in Figure 3; modes with $0 \leqslant \ell \leqslant 4$ were considered.

The measured amplitude ratios and phase shifts are consistent with the interpretation that all oscillation frequencies are caused by modes with $\ell \leqslant 2$; theoretical results for modes with higher $\ell$ are off scale in Figure 3. This corroborates the identification obtained in Figure 2.

Together with our ground-based multicolor photometry, our radial velocities can be used to constrain nonadiabatic pulsation theory, as empirical determinations of the $f$ parameter used to describe the bolometric flux amplitude depending on the surface displacement can be made. In turn, this can be used to choose the most suitable opacities for model calculations (DaszyńskaDaszkiewicz et al. 2005).

Figure 4 shows a comparison of theoretically predicted gmode frequencies of our $8.5 M_{\odot}$ model with the observations. The frequency separations of the g modes are well explained by this model: five of the six observed modes may form a sequence of consecutive radial overtones of $\ell=1$ modes. The sixth mode would then be $\ell=2$. The $\ell=1$ modes are stable; a final seismic model must explain their excitation.

We conclude that $\gamma$ Peg presents sufficient information to carry out detailed seismic modeling of a hybrid pulsator for the first time. The eight $\beta$ Cephei pulsation frequencies

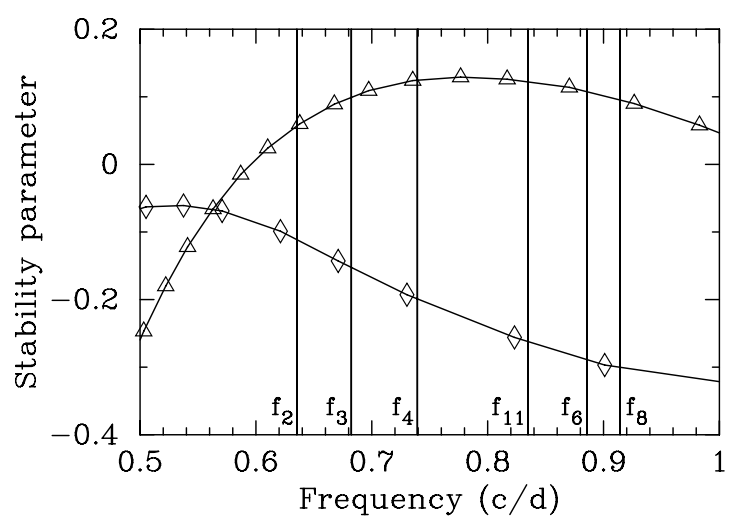

Figure 4. Comparison of theoretically predicted g-mode pulsation frequencies for an $8.5 M_{\odot}$ model of $\gamma$ Peg with the observed frequencies. As ordinate the stability parameter $\eta$ is used; if it is larger than zero, the corresponding mode is excited in the model. Diamonds represent dipole modes and triangles stand for quadrupole modes. The vertical lines are drawn at and identified with the observed frequencies.

restrict the possible models and their parameters considerably. The observed amplitude ratios and phase shifts between the radial velocities and photometric data provide clues toward the opacities to be used. Theoretical models constrained by such a large set of observables must also reproduce the high-order g modes and the excited frequency domains. The present study is a demonstration of the value of combining space photometry with ground-based spectroscopy of bright stars.

This work has been supported by the Austrian Fonds zur Förderung der wissenschaftlichen Forschung under grant P20526-N16. A.F.J.M. is grateful for financial support from NSERC (Canada) and FQRNT (Quebec). We thank Frank Fekel of Tennessee State University for providing the list of photospheric lines we used for extracting velocities from the TSU spectra. We are grateful to Ramotholo Sefako for organizing the SAAO observations.

\section{Facilities: TSU:AST, MOST, TLS}

\section{REFERENCES}

Asplund, M., Grevesse, N., Sauval, A. J., Allende Prieto, C., \& Kiselman, D. 2004, A\&A, 417, 751

Butkovskaya, V. V., \& Plachinda, S. I. 2007, A\&A, 469, 1069

Chapellier, E., Le Contel, D., Le Contel, J.-M., Mathias, P., \& Valtier, J.-C. 2006, A\&A, 448, 697

Daszyńska-Daszkiewicz, J., \& Niemczura, E. 2005, A\&A, 433, 431

Daszyńska-Daszkiewicz, J., Pamyatnykh, A. A., \& Dziembowski, W. A. 2005, A\&A, 441, 641

Dziembowski, W. A., \& Pamyatnykh, A. A. 2008, MNRAS, 385, 2061

Eaton, J. A., \& Williamson, M. H. 2007, PASP, 119, 886

Handler, G. 2009, MNRAS, in press, arXiv:0904.4859

Harmanec, P., Koubsky, P., Krpata, J., \& Zdarsky, F. 1979, IBVS, 1590

Kurucz, R. L. 2004, http://kurucz.harvard.edu

Lenz, P., \& Breger, M. 2005, Commun. Asteroseismol., 146, 53

McNamara, D. H. 1953, PASP, 65, 144

Montgomery, M. H., \& O’Donoghue, D. 1999, Delta Scuti Star Newsletter, 13, 28

Morel, T., Butler, K., Aerts, C., Neiner, C., \& Briquet, M. 2006, A\&A, 457, 651 Pamyatnykh, A. A., Dziembowski, W. A., Handler, G., \& Pikall, H. 1998, A\&A, 333,141

Pamyatnykh, A. A., Handler, G., \& Dziembowski, W. A. 2004, MNRAS, 350, 1022

Porter, J. M. 1996, MNRAS, 280, L31

Reegen, P., et al. 2006, MNRAS, 367, 1417

Telting, J. H., Schrijvers, C., Ilyin, I. V., Uytterhoeven, K., De Ridder, J., Aerts, C., \& Henrichs, H. F. 2006, A\&A, 452, 945

Walker, G., et al. 2003, PASP, 115, 1023 